ARTICLE

\title{
Exciton-acoustic phonon coupling revealed by resonant excitation of single perovskite nanocrystals
}

Yan Lv ${ }^{1,5}$, Chunyang Yin ${ }^{1,5}$, Chunfeng Zhang (1) ${ }^{1}$, Xiaoyong Wang (1) ${ }^{1 凶}$, Zhi-Gang Yu (i) ${ }^{2,3 凶} \&$ Min Xiao (i) $1,4 \bowtie$

\begin{abstract}
Single perovskite nanocrystals have attracted great research attention very recently due to their potential quantum-information applications, which critically depend on the development of powerful optical techniques to resolve delicate exciton photophysics. Here we have realized resonant and near-resonant excitations of single perovskite $\mathrm{CsPbl}_{3}$ nanocrystals, with the scattered laser light contributing to only $\sim 10 \%$ of the total collected signals. This allows us to estimate an ultranarrow photoluminescence excitation linewidth of $\sim 11.32 \mu \mathrm{eV}$ for the emission state of a single $\mathrm{CsPb}_{3}$ nanocrystal, corresponding to an exciton dephasing time of $\sim 116.29$ ps. Meanwhile, size-quantized acoustic phonons can be resolved from a single $\mathrm{CsPbl}_{3}$ nanocrystal, whose coupling with the exciton is proposed to arise from the piezoelectric potential. The ability to collect resonance fluorescence from single $\mathrm{CsPb}_{3}$ nanocrystals, with the subsequent revelation of exciton-acoustic phonon coupling, has marked a critical step towards their steady advancement into superior quantum-light sources.
\end{abstract}

\footnotetext{
${ }^{1}$ National Laboratory of Solid State Microstructures, School of Physics, and Collaborative Innovation Center of Advanced Microstructures, Nanjing University, Nanjing 210093, China. ${ }^{2}$ ISP/Applied Sciences Laboratory, Washington State University, Spokane, WA 99210, USA. ${ }^{3}$ Department of Physics and Astronomy, Washington State University, Pullman, WA 99164, USA. ${ }^{4}$ Department of Physics, University of Arkansas, Fayetteville, AR 72701, USA. ${ }^{5}$ These

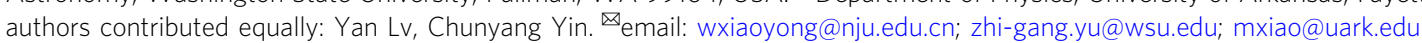


S emiconductor perovskite nanocrystals (NCs) have attracted great research attention in the single-particle optical studies since their first successful synthesis in 2015 (ref. ${ }^{1}$ ), with the subsequent observations of single-photon emission ${ }^{2,3}$, suppressed photoluminescence (PL) blinking and spectral diffusion ${ }^{4}$, and stable exciton fine structures ${ }^{5,6}$. By means of photon-correlation Fourier spectroscopy, the PL linewidth was measured to be $\sim 17.0 \mu \mathrm{eV}$ for the emission-state excitons of single $\mathrm{CsPbBr}_{3} \mathrm{NCs}^{7}$, while the exciton dephasing time of the absorption state could be longer than $\sim 10 \mathrm{ps}$ in single $\mathrm{CsPbI}_{3} \mathrm{NCs}$ based on the quantum interference measurement ${ }^{8}$. These coherent optical properties, which are rarely achievable in traditional colloidal NCs despite several decades of active pursuits, promise great potential of single perovskite NCs in quantum-information applications. In the aforementioned pioneering works ${ }^{7,8}$, a single perovskite NC was first excited into the absorption state with the coherent information being extracted next from the emission state, which would cause not only linewidth broadening to reduce the exciton coherence ${ }^{9,10}$ but also timing jitter in the photon generation and emission events ${ }^{11}$. To circumvent such undesired situations in the coherent optical studies of single perovskite NCs, it is imperative to realize resonant excitation of the emission state. This is analogous to the historical development of single epitaxial quantum dots (QDs), whose routine demonstrations of Mollow-triplet spectra $^{12}$ and indistinguishable single photons ${ }^{13}$ are critically dependent on the ability to collect the resonance fluorescence ${ }^{14}$.

Even under resonant excitation of a single epitaxial QD, it is still possible for the emission state to be disturbed by environmental spin and charge fluctuations ${ }^{15,16}$, as well as lattice vibrations of the acoustic-phonon modes ${ }^{16-18}$. Specifically, the exciton-acoustic phonon coupling could induce a broad sideband around the emission state, which fundamentally determines the upper limits that can be achieved for the lifetime of exciton coherence and the degree of photon indistinguishability ${ }^{19,20}$. The soft ionic lattice of semiconductor perovskites is featured with a strong anharmonicity ${ }^{21-23}$ to bring about low-energy and short-lived acoustic phonon modes ${ }^{23-27}$, with the accompanied low thermal conductivity ${ }^{28,29}$ and strong acoustic-optical phonon up-conversion ${ }^{30}$. These acoustic phonons can significantly influence the carrier transport and relaxation dynamics of semiconductor perovskites at the cryogenic temperatures. In contrast to the optical phonons that have been widely studied in the literature ${ }^{31,32}$, the acoustic phonons are yet to be experimentally detected in single perovskite NCs, let alone their possible influences on the exciton photophysical properties.

By adopting an orthogonal polarization geometry for laser excitation and PL collection, we show here that resonance fluorescence can be collected from single perovskite $\mathrm{CsPb}_{3} \mathrm{NCs}$ at the cryogenic temperature, with the residual contribution of scattered laser light being as low as $\sim 10 \%$. This allows us to resolve an ultranarrow PL excitation linewidth of $\sim 11.32 \mu \mathrm{eV}$ for the emission state of a single $\mathrm{CsPb}_{3} \mathrm{NC}$, corresponding to a dephasing time of $\sim 116.29 \mathrm{ps}$ for the band-edge excitons. We further demonstrate that a single $\mathrm{CsPbI}_{3} \mathrm{NC}$ can be efficiently excited when the laser energy is tuned across hundreds of $\mu \mathrm{eV}$ both above and below its emission state, which unambiguously confirms the participation of continuous acoustic phonons in the exciton generation processes. Moreover, a size-quantized acoustic-phonon mode is revealed under both near-resonant and resonant excitations of single $\mathrm{CsPbI}_{3} \mathrm{NCs}$, whose energy changes from $\sim 150$ to $\sim 180 \mu \mathrm{eV}$ with increase of the exciton's emission energy from $\sim 1.70$ to $\sim 1.73 \mathrm{eV}$ due to the reduction of $\mathrm{NC}$ size.

\section{Results}

Chemical synthesis and optical setup. The perovskite $\mathrm{CsPbI}_{3} \mathrm{NCs}$ are synthesized according to a standard hot-injection method $^{5}$ (see
Supplementary Methods in the Supplementary Information) with a cubic edge length of $\sim 9.31 \pm 0.68 \mathrm{~nm}$ (see the transmission electron microscopy image in Supplementary Fig. 1). Meanwhile, their longterm stability is obtained by the addition of tri-octylphosphine ligands in the post-synthesis treatment ${ }^{33}$. One drop of the diluted NC solution is spin-coated onto a fused silica substrate, which is then attached to the cold finger of a helium-free cryostat for the optical studies of single $\mathrm{CsPb}_{3} \mathrm{NCs}$. The experimental setup is schematically shown in Supplementary Fig. 2 (see Supplementary Methods in the Supplementary Information), where a He-Ne laser $(\sim 1.96 \mathrm{eV})$ and a tunable diode laser $(\sim 1.687-1.739 \mathrm{eV})$ both operated at the continuous-wave mode are employed for the above-bandgap and resonant/near-resonant excitations, respectively. For the purpose of observing resonance fluorescence from a single $\mathrm{CsPb}_{3} \mathrm{NC}$, two Glan-Thompson polarizers with orthogonal transmission axes are inserted into the laser excitation and PL collection paths, respectively, along with a quarter-wave plate to correct the birefringence effect caused by the relevant optical components ${ }^{20,34}$ (see Supplementary Fig. 3). Unless otherwise specified in the text, all the optical measurements are performed at the cryogenic temperature of $3 \mathrm{~K}$, and the laser excitation power is normally set at $\sim 100-500 \mathrm{nW}$ so that the $\mathrm{PL}$ intensity of a single $\mathrm{CsPbI}_{3} \mathrm{NC}$ is not saturated to minimize the possibility of generating multiple excitons (see Supplementary Fig. 4). Within this range of laser excitation power, all the single $\mathrm{CsPbI}_{3} \mathrm{NCs}$ studied in our experiment possess a very good chemical stability without suffering from the photo-bleaching effect during the measurement time as long as $15 \mathrm{~min}$.

Resonance fluorescence of single $\mathrm{CsPbI}_{3}$ NCs. In Fig. 1a, we plot the time-dependent PL spectral image of a representative single $\mathrm{CsPbI}_{3} \mathrm{NC}_{\text {excited at }} 1.96 \mathrm{eV}$, where the doublet PL peaks from the single-exciton fine structure ${ }^{5,6}$ can be clearly resolved with orthogonal linear polarizations. By summing over the $100 \mathrm{PL}$ spectra each acquired with an integration time of $5 \mathrm{~s}$ for the construction of this image, we show in Fig. 1b (top panel) that two sets of doublet PL peaks from longitudinal-optical (LO) phonons emerge from the background level in addition to the single-exciton one. These two LO phonon modes should arise from the $\mathrm{Pb}-\mathrm{I}-\mathrm{Pb}$ bending motions with the respective energies of $\sim 3.29$ and $\sim 5.29 \mathrm{meV}$, as revealed previously from the Raman and neutron scattering measurements ${ }^{25,28}$. In the bottom panel of Fig. 1b, by tuning the diode laser energy to either of the doublet peak positions, we further plot two PL spectra each obtained with an integration time of $1 \mathrm{~s}$. Compared with the $1.96 \mathrm{eV}$ excitation at the same laser power, PL intensities of the single-exciton doublet peaks are both enhanced under resonant excitations by almost two orders of magnitude, and the two LO phonon modes can be more easily resolved owing to the significantly-reduced background noise. To estimate the residual contribution of scattered laser light to the resonance fluorescence of the singleexciton doublet peaks (Fig. 1b, bottom panel), we select within the laser spot one blank area and another area with a single $\mathrm{CsPbI}_{3}$ NC whose optical signals can be simultaneously collected by the CCD camera for the comparison purpose.

As shown in Fig. 2a for the blank area (top panel), when the laser energy is scanned over time from $\sim 1.720-1.717 \mathrm{eV}$ with a step resolution of $\sim 7.35 \mu \mathrm{eV} / \mathrm{s}$, the monitored intensity of the moving laser peak on the CCD camara shows small fluctuations around a constant value. For the single-NC area in the middle panel of Fig. 2a, there appear two batches of intensity spikes during $\sim 430-510$ and $\sim 580-660 \mathrm{~s}$ when the laser energy is being tuned to the doublet-peak positions with the higher and lower energies, respectively. This indicates that resonance fluorescence of a single $\mathrm{CsPbI}_{3} \mathrm{NC}$ has been successfully collected during these two time periods, which is supported by the similar trend observed in 
a

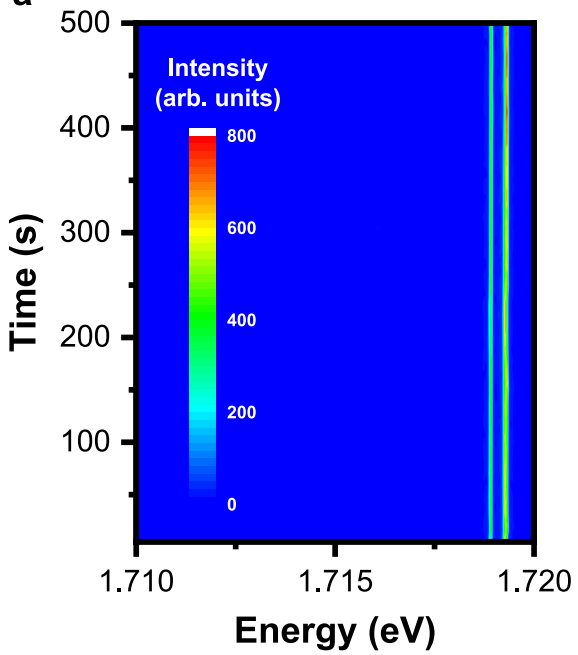

b

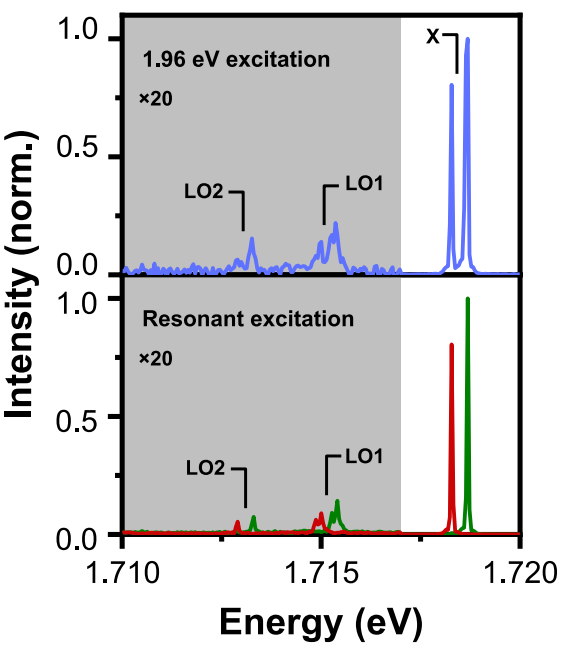

Fig. 1 Fundamental optical properties of a single $\mathbf{C s}_{\mathbf{P b}} \mathbf{3} \mathbf{~ N C}$. a Time-dependent PL spectral image constructed from $100 \mathrm{PL}$ spectra each acquired with an integration time of $5 \mathrm{~s}$ under the $1.96 \mathrm{eV}$ laser excitation. $\mathbf{b}$ (top panel) PL spectrum plotted by summing over the $100 \mathrm{PL}$ spectra used in a to construct the image. (bottom panel) Two PL spectra measured with the laser excitation energies being resonant with the higher- and lower-energy PL peaks, respectively, both using an integration time of $1 \mathrm{~s}$. In $\mathbf{b}, \mathrm{PL}$ intensities in the shaded areas are magnified by 20 times, while $\mathrm{X}$ and LO1/LO2 denote the single-exciton doublet peaks and the two longitudinal-optical phonon modes, respectively.

the bottom panel of Fig. 2a from the integrated PL intensity of the two LO phonon peaks. Owing to the mode-hopping effect of the diode laser energy (Fig. 2a, top panel), each of the doublet peaks can be excited multiple times during the scanning, yielding several intensity spikes instead of a single one (Fig. $2 \mathrm{a}$, middle and bottom panels). This mode-hopping effect and the accompanied variation in the laser power, as reflected in the fluctuating background signals in the top and middle panels of Fig. $2 a$, give rise to the intensity differences among these spikes. By comparing the maximum intensity of these spikes with that of the background signal (Fig. 2a, middle panel), we can estimate that the contribution of scattered laser light to each of the resonantly-excited PL spectra in Fig. 1b (bottom panel) is as low as $\sim 10 \%$.

On the same single $\mathrm{CsPbI}_{3} \mathrm{NC}$, we next tune the laser energy across a very small range around either of the doublet peaks, so that their corresponding changes of the PL intensities can be smoothly measured without suffering from the mode-hopping effect. As shown in Fig. 2b for the lower-energy peak, the PL excitation spectrum can be fitted by a Lorentzian function with a linewidth of $\sim 11.32 \mu \mathrm{eV}$. This kind of PL excitation measurement has been performed for a total of 21 single $\mathrm{CsPbI}_{3} \mathrm{NCs}$ in our experiment, and an average linewidth of $\sim 17.62 \mu \mathrm{eV}$ can be calculated from the distribution histogram shown in Fig. 2c. For the single $\mathrm{CsPbI}_{3} \mathrm{NC}$ with the PL excitation linewidth of $\sim 11.32 \mu \mathrm{eV}$ (Fig. $2 \mathrm{~b}$ ), it can be judged from the time-dependent PL spectral image in Fig. 1a that there exists no spectral diffusion within our system resolution of $\sim 100 \mu \mathrm{eV}$. In contrast, for single $\mathrm{CsPbI}_{3} \quad \mathrm{NCs}$ with broader linewidths such as $\sim 17.40 \mu \mathrm{eV}$ (Supplementary Fig. 5a), the single-exciton doublet peaks suffer obviously from the spectral diffusion effect with the occasional transition to a charged-exciton peak (Supplementary Fig. 5b). The above observations suggest that charge fluctuations could be one likely cause to broaden the PL excitation linewidth of a single $\mathrm{CsPbI}_{3} \mathrm{NC}$, which might be also influenced by the excitonacoustic phonon coupling effect as discussed below.

Exciton-acoustic phonon coupling. In Fig. 3a, we construct a 2D image for a single $\mathrm{CsPbI}_{3} \mathrm{NC}$ to show in detail how the PL intensities of its doublet peaks evolve within a larger range of laser scanning energies from $\sim 1.7175-1.7195 \mathrm{eV}$. In addition to the resonant enhancement, each of the doublet peaks can still be effectively excited when the laser energy is detuned to its blue or red side by hundreds of $\mu \mathrm{eV}$. As can be seen in the top panel of Fig. $3 b$ with a variation of the detuned laser energy, PL intensity of the lower-energy peak rises from $-250 \mu \mathrm{eV}$ to reach a maximum at $0 \mu \mathrm{eV}$ and then decays to the background level at $350 \mu \mathrm{eV}$. This broad PL excitation spectrum is associated with an asymmetric lineshape, showing that an excitation efficiency larger than $\sim 16.6 \%$ can be achieved by the blue-detuned laser as compared to that of the resonant excitation. When the sample temperature is increased to $10 \mathrm{~K}$ (Fig. $3 \mathrm{~b}$, bottom panel), the lower-energy peak of a single $\mathrm{CsPbI}_{3} \mathrm{NC}$ can now be excited within a larger energy range from -450 to $600 \mu \mathrm{eV}$, with the excitation efficiency being elevated to more than $\sim 37.1 \%$ for the blue-detuned laser. The above results strongly suggest the involvement of acoustic phonons with continuous energies in the exciton down- and up-conversion processes under the blue- and red-detuned laser excitations, respectively, as reported previously from similar optical studies of single epitaxial InGaAs QDs ${ }^{10}$.

As shown in the inset of Fig. 3b (top panel), two side peaks can be additionally resolved at about -190 and $190 \mu \mathrm{eV}$ from the PL excitation spectrum that has been replotted for an amplified view of the low-signal region. In fact, these two side peaks also appear in the PL spectral image of Fig. 3a when either of the doublet peaks is being resonantly excited. In Fig. $4 \mathrm{a}$ and b, we further plot two resonantly-excited PL spectra for the higher- and lowerenergy emission states of another single $\mathrm{CsPbI}_{3} \mathrm{NC}$, respectively. By fitting each PL spectrum with multiple Gaussian functions, the two side peaks are estimated to be $\sim 155 \mu \mathrm{eV}$ below and above the central peak with an intensity ratio of $\sim 1.50$. Such set of downand up-converted side peaks has been universally observed in the 24 single $\mathrm{CsPbI}_{3} \mathrm{NCs}$ studied in our experiment, from which an average intensity ratio of $\sim 1.47 \pm 0.23$ can be obtained. Meanwhile, when the emission energy of the central PL peak increases from $\sim 1.70-1.73 \mathrm{eV}$ in different single $\mathrm{CsPbI}_{3} \mathrm{NCs}$, each side peak is detuned away from it with an increasing energy separation from $\sim 150-180 \mu \mathrm{eV}$ (Supplementary Fig. 8). Since this energy separation is independent of the laser excitation power (Supplementary Fig. 9), the Mollow-triplet origin $^{12}$ can be safely 

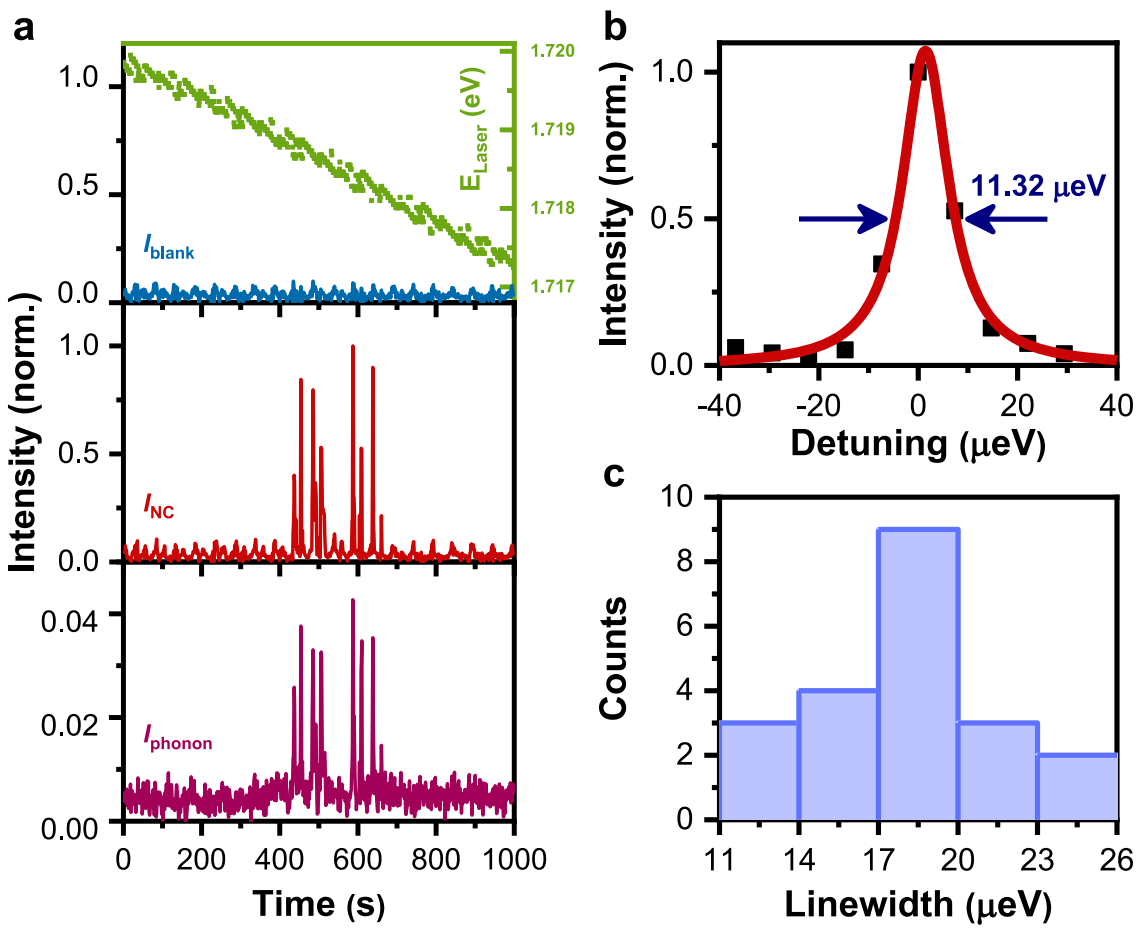

Fig. 2 Resonance fluorescence from a single $\mathbf{C s P b l}_{\mathbf{3}} \mathbf{N C}$. a (top panel) Time-dependent energy and intensity of the moving laser peak scanned from $\sim 1.720-1.717 \mathrm{eV}$ on the blank sample area. (middle panel) The same intensity measurement on the single-NC sample area. (bottom panel) Integrated PL intensity of the two LO phonon modes plotted as a function of the measurement time. $\mathbf{b}$ PL intensity of the lower-energy peak plotted as a function of the detuned laser energy and fitted with a linewidth of $\sim 11.32 \mu \mathrm{eV}$ for this single $\mathrm{CsPbl}_{3} \mathrm{NC}$. c Statistical histogram showing the linewidth distribution of the PL excitation spectra measured for the emission states of 21 single $\mathrm{CsPb}_{3} \mathrm{NCs}$.
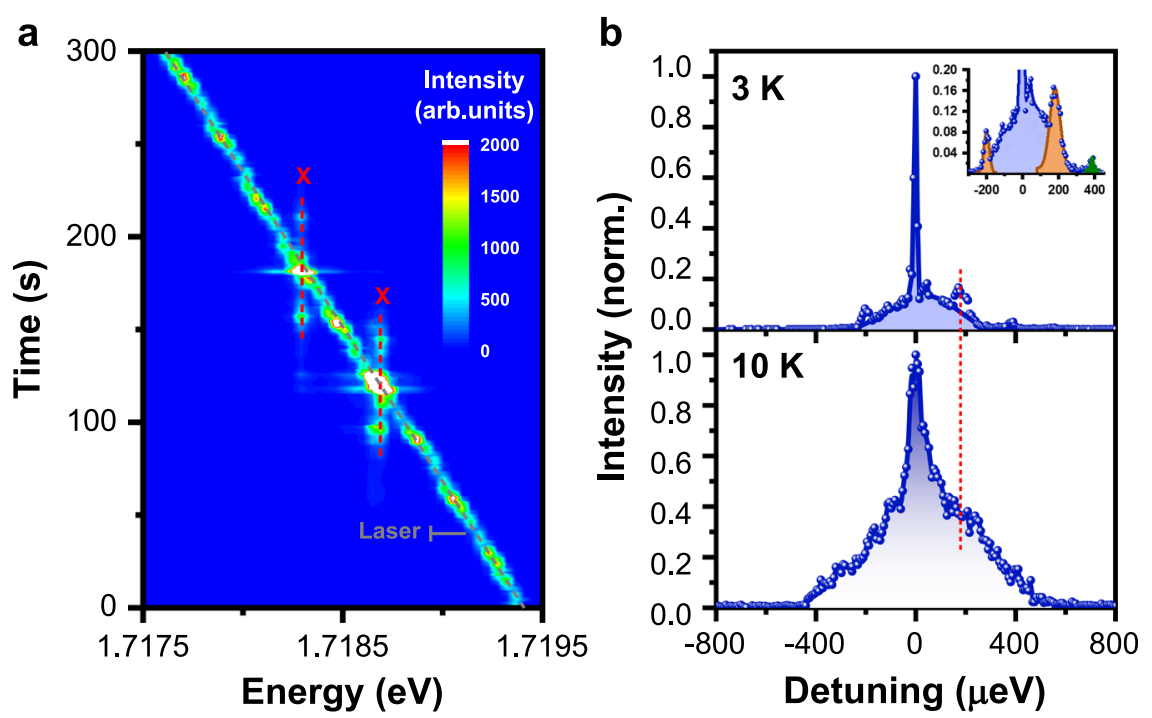

Fig. 3 Continuous acoustic phonons in a single $\mathbf{C s P b l}_{\mathbf{3}} \mathbf{N C}$. a 2D image showing intensity variations of the doublet PL peaks with the laser scanning energies from $1.7175-1.7195 \mathrm{eV}$. Here $\mathrm{X}$ denotes the energy position for either of the doublet PL peaks. $\mathbf{b}$ (top panel) PL intensity of the lower-energy peak plotted as a function of the detuned laser energy at $3 \mathrm{~K}$. Inset: Amplified view of the low-signal region. (bottom panel) PL intensity of the lower-energy peak plotted as a function of the detuned laser energy at $10 \mathrm{~K}$. The dotted line marks the energy position where the phonon-assisted excitation efficiencies of $\sim 16.6$ and $\sim 37.1 \%$ can be achieved at 3 and $10 \mathrm{~K}$, respectively, as compared to that of the resonant excitation at $0 \mu \mathrm{eV}$. See Supplementary Fig. 6 and related discussions in the Supplementary Information for theoretical fits of the PL excitation spectra measured at 3 and $10 \mathrm{~K}$.

excluded. Nor could they be attributed to the exciton fine structures and LO phonon modes, which have been well resolved in our experiment. Then the two side peaks should correspond to a size-quantized acoustic-phonon mode arising from the spatial confinement of lattice vibrations ${ }^{35}$.
Although these size-quantized acoustic phonons can be easily detected under resonant excitation, they only show up in the PL spectra of a limited number of single $\mathrm{CsPbI}_{3} \mathrm{NCs}$ excited above the bandgap at $1.96 \mathrm{eV}$ (Supplementary Fig. 10) due to the poor signal-to-noise ratio mentioned earlier in the text. 

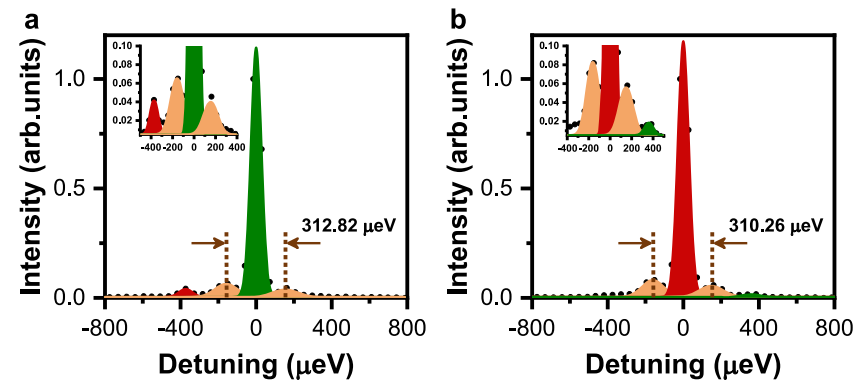

Fig. 4 Size-quantized acoustic phonons in a single $\mathrm{CsPbl}_{3} \mathbf{N C}$. a

Resonantly-excited higher-energy peak (green), the associated side peaks from size-quantized acoustic photons (orange), and the down-converted lower-energy peak (red). Inset: Amplified view of the low-signal region. See Supplementary Fig. 7 and related discussions in the Supplementary Information for theoretical fit of this resonantly-excited PL spectrum. b Resonantly-excited lower-energy peak (red), the associated side peaks from size-quantized acoustic photons (orange), and the up-converted higher-energy peak (green). Inset: Amplified view of the low-signal region.

In Supplementary Fig. 11, we plot the PL spectra of two such single $\mathrm{CsPbI}_{3} \mathrm{NCs}$ excited at $1.96 \mathrm{eV}$ with the emission energies of $\sim 1.7332$ and $\sim 1.7464 \mathrm{eV}$, respectively, and the associated energies of their size-quantized acoustic phonons are estimated to be $\sim 210$ and $\sim 260 \mu \mathrm{eV}$, respectively. The above observations thus further confirm the increasing trend of the size-quantized acoustic-phonon energies with the increasing $\mathrm{NC}$ emission energies, which deserves intensive future studies with different $\mathrm{CsPbI}_{3} \mathrm{NC}$ samples and tunable diode lasers other than those employed in the current experiment.

The influences of acoustic phonons on the exciton emission linewidths and relaxation dynamics were previously reported in traditional colloidal $\mathrm{NCs}^{36-43}$, however, this is the first time that they have been detected directly from the optical studies of single perovskite NCs. In general, the exciton-acoustic phonon coupling can originate from either the short-range deformational potential, or the long-range piezoelectric potential if the material lacks an inversion symmetry ${ }^{35}$. In the former case, the coupling is due mainly to the longitudinal acoustic (LA) phonons when the conduction and valence bands are approximately isotropic as in the perovskite $\mathrm{CsPb}_{3}$ material. Whereas in the latter case, both LA and transverse acoustic (TA) phonons can be involved in the exciton-acoustic phonon coupling. In perovskite materials, the piezoelectric coupling between excitons and TA phonons is particularly strong (see theoretical calculations in the Supplementary Information). For a small phonon wave vector $q$, the deformational and piezoelectric coupling strengths are $V_{d}(q) \sim$ $q^{1 / 2}$ and $V_{p}(q) \sim q^{-1 / 2}$, respectively, implying that the piezoelectric coupling would reach a maximum for the smallest wave vector allowed by a finite-sized NC. The small- $q$ acoustic phonons, with their wavelengths (approximately the NC size) being much longer than the lattice constant, can be described by the elastic continuum theory, and their wave velocity and polarization along a given direction can be obtained by solving the Christoffel equation ${ }^{44}$.

In particular, for the acoustic wave propagating along the symmetry axes such as [100], there exist one LA mode with the velocity $v_{l}=\left(C_{11} / \rho\right)^{1 / 2}$ and two TA modes with the velocity $v_{t}=\left(C_{44} / \rho\right)^{1 / 2}$, where $C_{11}$ is the axial compression modulus, $C_{44}$ is the shear modulus, and $\rho$ is the mass density. Using the theoretical values of $C_{11}=34.23 \mathrm{GPa}, C_{44}=3.24 \mathrm{GPa}$ and $\rho=5.39 \mathrm{~g} / \mathrm{cm}^{3}$ reported in the literature for perovskite materials 45,46 , we can obtain the velocities of $v_{l}=2.52 \times 10^{3}$ and $v_{t}=0.78 \times 10^{3} \mathrm{~m} / \mathrm{s}$, respectively. Since the smallest wave vector along the symmetry axis in a single $\mathrm{CsPbI}_{3} \mathrm{NC}$ is $q=\pi / L$ with $L$ being the edge length, the corresponding energies of the LA and TA modes are $\hbar \omega_{l}=\hbar v_{l} q=560 \mu \mathrm{eV}$ and $\hbar \omega_{t}=\hbar v_{t} q=172 \mu \mathrm{eV}$, respectively. Thus, the energy range of $\sim 150-180 \mu \mathrm{eV}$ (Supplementary Fig. 8) obtained for the size-quantized acoustic phonons should be related to the TA modes, which couple strongly with the excitons via the piezoelectric potential (see theoretical calculations of the Supplementary Information). On the other hand, the coupling of excitons with continuous acoustic phonons, as observed here in single $\mathrm{CsPbI}_{3} \mathrm{NCs}$ (Fig. 3b) and previously in many other inorganic nanostructures ${ }^{47}$, could proceed through weaker piezoelectric and deformational potentials that couple to LA phonons.

Influence of acoustic phonons on exciton transitions. When the higher-energy peak of a single $\mathrm{CsPbI}_{3} \mathrm{NC}$ is being resonantly excited, it can be seen from the inset of Fig. 4a that the lowerenergy peak $\sim 370 \mu \mathrm{eV}$ below also shows up in addition to the size-quantized acoustic-phonon modes. When the laser energy is off this resonant position by only several $\mu \mathrm{eV}$, PL intensity of the lower-energy peak is reduced almost completely to the background level. This suggests that the lower-energy state is not populated directly by the laser excitation, but instead via exciton relaxation from the higher-energy state by emitting acoustic phonons. However, this exciton relaxation process is extremely inefficient, as reflected by the intensity ratio of $\sim 0.040$ between the lower- and higher-energy PL peaks. Similarly shown in the inset of Fig. 4b, the higher-energy peak can also be detected when the lower-energy peak is being resonantly excited, albeit with a much smaller ratio of $\sim 0.015$ between their PL intensities in this exciton up-conversion process. Based on the fact that the two emission states have an energy-level splitting of $\sim 370 \mu \mathrm{eV}$, which is obviously larger than the maximum acoustic phonon energy of $\sim 350 \mu \mathrm{eV}$ detected in a single $\mathrm{CsPbI}_{3} \mathrm{NC}$, the above observations can be understood via a higher-order multiple phonon process with the piezoelectric coupling. These two emission states are associated with bright excitons of a single $\mathrm{CsPbI}_{3} \mathrm{NC}^{5}$, and it was revealed very recently from a magneto-optical study that they are several meV higher in energy than the dark-exciton state ${ }^{48}$. This makes it even more difficult for the bright excitons to relax into the dark state, which naturally explains why the emission states of a single $\mathrm{CsPbI}_{3} \mathrm{NC}$ are highly luminescent, without seeking the Rashba effect to place the dark state in the highest-energy position $^{6}$ or a two-optical phonon Raman scattering process for the mixing of bright and dark states ${ }^{31}$. It should be noted that the exciton transition process between the bright states could be thermally enhanced due to the availability of more acoustic phonons, as is shown in Supplementary Fig. 12 from the resonantly-excited PL spectra measured for a single $\mathrm{CsPbI}_{3} \mathrm{NC}$ at different temperatures.

\section{Discussion}

To summarize, we have realized resonant and near-resonant excitations of single perovskite $\mathrm{CsPb}_{3} \mathrm{NCs}$, with the scattered laser light accounting for as low as $\sim 10 \%$ of the total collected signals. Further reduction of the residual laser light is possible when the excitation beam is incident on the sample surface with a tilted angle, instead of passing through the objective confocally in our experiment. However, the current optical setup has already allowed us to probe the continuous and size-quantized acousticphonon modes with a high signal-to-noise ratio, whose energies are distributed within the ranges of $0-350$ and $150-180 \mu \mathrm{eV}$, respectively. These acoustic phonons are indispensable in assisting the detuned laser excitation of a single $\mathrm{CsPbI}_{3} \mathrm{NC}$, allowing us to resolve an ultranarrow PL excitation linewidth of 
$\sim 11.32 \mu \mathrm{eV}$ that can be regarded as an upper limit for the intrinsic emission-state PL linewidth. The transformed exciton dephasing time of $\sim 116.29$ ps is almost one order of magnitude shorter than the exciton radiative lifetime of $\sim 1 \mathrm{~ns}^{5}$, the latter of which can be surely accelerated in future works by coupling a single $\mathrm{CsPbI}_{3} \mathrm{NC}$ to a plasmonic nanocavity ${ }^{49}$ or a dielectric waveguide $^{50}$ for the generation of indistinguishable single photons. Our results indicate that, so long as the acoustic-phonon energies are smaller than the fine energy-level splittings, thermal mixing among the bright and dark states is greatly minimized to ensure a long exciton dephasing time. Overall, the ability to collect resonance fluorescence and the revelation of acoustic-phonon modes as well as their role in the exciton dephasing process represent a critical step in the development of single $\mathrm{CsPb}_{3} \mathrm{NCs}$ as a superior singlephoton source for quantum-information applications.

\section{Data availability}

The data supporting the findings of this study are available from the corresponding authors upon request.

Received: 14 December 2020; Accepted: 16 March 2021; Published online: 13 April 2021

\section{References}

1. Protesescu, L. et al. Nanocrystals of cesium lead halide perovskites $\left(\mathrm{CsPbX}_{3}, \mathrm{X}\right.$ $=\mathrm{Cl}, \mathrm{Br}$, and I): novel optoelectronic materials showing bright emission with wide color gamut. Nano Lett. 15, 3692-3696 (2015).

2. Hu, F. et al. Superior optical properties of perovskite nanocrystals as single photon emitters. ACS Nano 9, 12410-12416 (2015).

3. Park, Y.-S., Guo, S., Makarov, N. S. \& Klimov, V. I. Room temperature singlephoton emission from individual perovskite quantum dots. ACS Nano $\mathbf{9}$, 10386-10393 (2015)

4. Hu, F. et al. Slow Auger recombination of charged excitons in nonblinking perovskite nanocrystals without spectral diffusion. Nano Lett. 16, 6425-6430 (2016).

5. Yin, C. et al. Bright-exciton fine-structure splittings in single perovskite nanocrystals. Phys. Rev. Lett. 119, 026401 (2017).

6. Becker, M. A. et al. Bright triplet excitons in caesium lead halide perovskites. Nature 553, 189-193 (2018).

7. Utzat, H. et al. Coherent single-photon emission from colloidal lead halide perovskite quantum dots. Science 363, 1068-1072 (2019).

8. Lv, Y. et al. Quantum interference in a single perovskite nanocrystal. Nano. Lett. 19, 4442-4447 (2019).

9. Bennett, A. J., Unitt, D. C., Shields, A. J., Atkinson, P. \& Ritchie, D. A. Influence of exciton dynamics on the interference of two photons from a microcavity single-photon source. Opt. Express 13, 7772-7778 (2005).

10. Weiler, S. et al. Phonon-assisted incoherent excitation of a quantum dot and its emission properties. Phys. Rev. B 86, 241304 (2012).

11. Kiraz, A., Atatüre, M. \& Imamoğlu, A. Quantum-dot single-photon sources: prospects for applications in linear optics quantum-information processing. Phys. Rev. A 69, 032305 (2004).

12. Flagg, E. B. et al. Resonantly driven coherent oscillations in a solid-state quantum emitter. Nat. Phys. 5, 203-207 (2009).

13. He, Y.-M. et al. On-demand semiconductor single-photon source with nearunity indistinguishability. Nat. Nanotechnol. 8, 213-217 (2013).

14. Muller, A. et al. Resonance fluorescence from a coherently driven semiconductor quantum dot in a cavity. Phys. Rev. Lett. 99, 187402 (2007).

15. Kuhlmann, A. V. et al. Charge noise and spin noise in a semiconductor quantum device. Nat. Phys. 9, 570-575 (2013).

16. Thoma, A. et al. Exploring dephasing of a solid-state quantum emitter via time- and temperature-dependent Hong-Ou-Mandel experiments. Phys. Rev. Lett. 116, 033601 (2016)

17. Ulrich, S. M. et al. Dephasing of triplet-sideband optical emission of a resonantly driven InAs/GaAs quantum dot inside a microcavity. Phys. Rev. Lett. 106, 247402 (2011)

18. Iles-Smith, J., McCutcheon, D. P. S., Nazir, A. \& Mørk, J. Phonon scattering inhibits simultaneous near-unity efficiency and indistinguishability in semiconductor single-photon sources. Nat. Photon 11, 521-526 (2017).

19. Brash, A. J. et al. Light scattering from solid-state quantum emitters: beyond the atomic picture. Phys. Rev. Lett. 123, 167403 (2019).
20. Koong, Z. X. et al. Fundamental limits to coherent photon generation with solid-state atomlike transitions. Phys. Rev. Lett. 123, 167402 (2019).

21. Marronnier, A. et al. Anharmonicity and disorder in the black phases of cesium lead iodide used for stable inorganic perovskite solar cells. ACS Nano 12, 3477-3486 (2018)

22. Whalley, L. D., Skelton, J. M., Frost, J. M. \& Walsh, A. Phonon anharmonicity, lifetimes, and thermal transport in $\mathrm{CH}_{3} \mathrm{NH}_{3} \mathrm{PbI}_{3}$ from many-body perturbation theory. Phys. Rev. B 94, 220301 (2016).

23. Yaffe, O. et al. Local polar fluctuations in lead halide perovskite crystals. Phys Rev. Lett. 118, 136001 (2017).

24. Even, J., Carignano, M. \& Katan, C. Molecular disorder and translation/ rotation coupling in the plastic crystal phase of hybrid perovskites. Nanoscale 8, 6222-6236 (2016).

25. Ferreira, A. C. et al. Direct evidence of weakly dispersed and strongly anharmonic optical phonons in hybrid perovskites. Commun. Phys. 3, 48 (2020).

26. Gold-Parker, A. et al. Acoustic phonon lifetimes limit thermal transport in methylammonium lead iodide. Proc. Natl. Acad. Sci. USA 115, 11905-11910 (2018).

27. Songvilay, M. et al. Lifetime-shortened acoustic phonons and static order at the Brillouin zone boundary in the organic-inorganic perovskite $\mathrm{CH}_{3} \mathrm{NH}_{3} \mathrm{PbCl}_{3}$. Phys. Rev. Mater. 2, 123601 (2018).

28. Li, B. et al. Polar rotor scattering as atomic-level origin of low mobility and thermal conductivity of perovskite $\mathrm{CH}_{3} \mathrm{NH}_{3} \mathrm{PbI}_{3}$. Nat. Commun. 8, 16086 (2017).

29. Ferreira, A. C. et al. Elastic softness of hybrid lead halide perovskites. Phys. Rev. Lett. 121, 085502 (2018)

30. Yang, J. et al. Acoustic-optical phonon up-conversion and hot-phonon bottleneck in lead-halide perovskites. Nat. Commun. 8, 14120 (2017).

31. Fu, M. et al. Unraveling exciton-phonon coupling in individual $\mathrm{FAPbI}_{3}$ nanocrystals emitting near-infrared single photons. Nat. Commun. 9, 3318 (2018).

32. Pfingsten, O. et al. Phonon interaction and phase transition in single formamidinium lead bromide quantum dots. Nano. Lett. 18, 4440-4446 (2018).

33. Lu, C. et al. Enhanced stabilization of inorganic cesium lead triiodide $\left(\mathrm{CsPb}_{3}\right)$ perovskite quantum dots with tri-octylphosphine. Nano Res. 11, 762-768 (2017).

34. Kuhlmann, A. V. et al. A dark-field microscope for background-free detection of resonance fluorescence from single semiconductor quantum dots operating in a set-and-forget mode. Rev. Sci. Instrum. 84, 073905 (2013).

35. Takagahara, T. Electron-phonon interactions and excitonic dephasing in semiconductor nanocrystals. Phys. Rev. Lett. 71, 3577-3580 (1993).

36. Krauss, T. D. \& Wise, F. W. Coherent acoustic phonons in a semiconductor quantum dot. Phys. Rev. Lett. 79, 5102-5105 (1997).

37. Cerullo, G., De Silvestri, S. \& Banin, U. Size-dependent dynamics of coherent acoustic phonons in nanocrystal quantum dots. Phys. Rev. B 60, 1928-1932 (1999).

38. Ikezawa, M., Okuno, T., Masumoto, Y. \& Lipovskii, A. A. Complementary detection of confined acoustic phonons in quantum dots by coherent phonon measurement and Raman scattering. Phys. Rev. B 64, 201315 (2001).

39. Palinginis, P., Tavenner, S., Lonergan, M. \& Wang, H. L. Spectral hole burning and zero phonon linewidth in semiconductor nanocrystals. Phys. Rev. B 67, 201307 (2003)

40. Chilla, G. et al. Direct observation of confined acoustic phonons in the photoluminescence spectra of a single CdSe-CdS-ZnS core-shell-shell nanocrystal. Phys. Rev. Lett. 100, 057403 (2008).

41. Werschler, F. et al. Coupling of excitons and discrete acoustic phonons in vibrationally isolated quantum emitters. Nano Lett. 16, 5861-5865 (2016).

42. Fernée, M. J. et al. Acoustic phonon contributions to the emission spectrum of single CdSe nanocrystals. J. Phys. Chem. C. 112, 1878-1884 (2008).

43. Fernée, M. J. et al. Magneto-optical properties of trions in non-blinking charged nanocrystals reveal an acoustic phonon bottleneck. Nat. Commun. 3, 1287 (2012)

44. Landau, L. D. \& Lifshits, E. M. Theory of Elasticity. Pergamon Press (1964).

45. Roknuzzaman, M., Ostrikov, K., Wang, H., Du, A. \& Tesfamichael, T. Towards lead-free perovskite photovoltaics and optoelectronics by ab-initio simulations. Sci. Rep. 7, 14025 (2017).

46. Stoumpos, C. C., Malliakas, C. D. \& Kanatzidis, M. G. Semiconducting tin and lead iodide perovskites with organic cations: phase transitions, high mobilities, and near-infrared photoluminescent properties. Inorg. Chem. 52, 9019-9038 (2015).

47. Besombes, L., Kheng, K., Marsal, L. \& Mariette, H. Acoustic phonon broadening mechanism in single quantum dot emission. Phys. Rev. B 63, 155307 (2001).

48. Tamarat, P. et al. The dark exciton ground state promotes photon-pair emission in individual perovskite nanocrystals. Nat. Commun. 11, 6001 (2020). 
49. Hoang, T. B., Akselrod, G. M. \& Mikkelsen, M. H. Ultrafast room-temperature single photon emission from quantum dots coupled to plasmonic nanocavities. Nano Lett. 16, 270-275 (2016).

50. Kolchin, P. et al. High Purcell factor due to coupling of a single emitter to a dielectric slot waveguide. Nano Lett. 15, 464-468 (2015).

\section{Acknowledgements}

This work is supported by the National Basic Research Program of China (2019YFA0308704 and 2017YFA0303700), the National Natural Science Foundation of China (No. 61974058), and the PAPD of Jiangsu Higher Education Institutions.

\section{Author contributions}

X.W., C.Z. and M.X. conceived and designed the experiments. Y.L. and C.Y. prepared the samples and performed the optical measurements. Z.Y. did the theoretical calculations. Y.L., C.Y. and X.W. analyzed the data. X.W., Z.Y., C.Z. and M.X. co-wrote the manuscript.

\section{Competing interests}

The authors declare no competing interests.

\section{Additional information}

Supplementary information The online version contains supplementary material available at https://doi.org/10.1038/s41467-021-22486-5.
Correspondence and requests for materials should be addressed to X.W., Z.-G.Y. or M.X.

Peer review information Nature Communications thanks the anonymous reviewer(s) for their contribution to the peer review of this work.

Reprints and permission information is available at http://www.nature.com/reprints

Publisher's note Springer Nature remains neutral with regard to jurisdictional claims in published maps and institutional affiliations.

(c) (i) Open Access This article is licensed under a Creative Commons Attribution 4.0 International License, which permits use, sharing, adaptation, distribution and reproduction in any medium or format, as long as you give appropriate credit to the original author(s) and the source, provide a link to the Creative Commons license, and indicate if changes were made. The images or other third party material in this article are included in the article's Creative Commons license, unless indicated otherwise in a credit line to the material. If material is not included in the article's Creative Commons license and your intended use is not permitted by statutory regulation or exceeds the permitted use, you will need to obtain permission directly from the copyright holder. To view a copy of this license, visit http://creativecommons.org/ licenses/by/4.0/.

(C) The Author(s) 2021 